\title{
Research on the Working Principle of Machine Vision and Its Application in Industrial Robot
}

\author{
Lei Chen ${ }^{1, a}$ \\ ${ }^{1}$ Jiangxi Vocational Techlege Of Industry \& Trade, Jiangxi, Nanchang, 330038 \\ a739960103@qq.com

\begin{abstract}
This paper mainly explores the application thinking and development of industrial robot technology from the perspective of machine. For the current development of industrial robot technology, if you want to explore and develop mechanical engineering technology from the mechanical market, you must recognize the limitations of working environment and working conditions and the major challenges to the operating system of industrial robot. Therefore, to improve the overall comprehensive efficiency and quality of modern industrial production, you must solve the technical development of industrial robot from all aspects, Let industrial robots enter the intelligent era.
\end{abstract}

Keywords: machine vision; Industrial robot; Modern automatic production; Application exploration

\section{机器视觉的工作原理与在工业机器人方面的应用研究 \\ 陈䂞 ${ }^{1, a}$}

\author{
${ }^{1}$ 江西工业贸易职业技术学院 江西 南昌 330038 \\ a739960103@qq.com
}

摘要:

本文主要探索在机器视角的观察下工业机器人技术方面的应用思考与发展。对于目前的工业机器人技术发展 而言, 想要从机械市场对机械工程技术进行探究和发展, 就必须认识到工作环境和工作条件的限制, 对于工 业机器人操作系统的重大挑战, 因此提高现代工业化生产的总体综合效率和质量, 就必须从各方面解决工业 机器人的技术发展, 让工业机器人进入智能化时代。

关键词: 机器视觉; 工业机器人；现代自动化生产; 应用探究

\section{1 机器视觉的工作原理及其与工业机器人的内 在联系}

\section{1 机器视觉的工作原理}

所谓的机器视觉系统主要就是将一种视觉传感 器装配在生产机器人身上, 通过将被检测目标的各 种情况进行电子图像绘图的一种电子图像技术, 往 往通过机械视角系统绘制的电子图像不能清晰的表 达产品的情况, 因此想要进一步的得到机械市场下 的检测目标个性的图像, 就必须通过专业的图像处 理系统将所检测的图像变成清晰的电子图像, 同时 在进一步的根据电子图像的色彩特征, 将亮度显示 的一些图形特点表现出来, 方便检测目标物的特征
分析和检测, 通过图像的结果来操纵机器人进行相 应的动作管理。

整个视觉传感系统分为三个部分, 分别是图像 的获取, 图像的处理和分析, 以及分析结果的输出 和显示。

对于工业机器人的领域而言, 视觉系统是一个 重要的研究方向, 随着信息化时代的来临科技的不 断发展。生产出来的机器视觉系统, 往往能够在计 算机技术和工程技术的加持下，能够达到人类眼睛 的部分功能, 将这些功能与工业机器人联系起来, 能够有效的将机器人工作过程中想要检测目标物, 进行图像信息检测，从而更好的提高工业机器人在 整个生产过程中对于检测物的准确性和高度定位性。 


\section{2 与工业机器人的内在联系}

现存的工业机器人主要应用在制造业生产检测 以及物流运输行业中。

在这些行业领域中, 对于工业机器人本身的操 作精密度就有着较高的要求, 所以机器视觉在工业 机器人的工作中能够有效的让机器人满足各类环境 的视觉工作, 并且能够有效的把工作生产中的效率 和精密度质量提升到一定的层次, 从而在未来的工 作发展过程中, 工业机器人通过视觉系统能够有效 地代替相关人员的工作，达到真正的生产自动化。

\section{2 机器视觉在工业机器人方面的应用}

机器视觉在工业机器人方面的应用中主要体现 在工业机器人零部件装配中的应用; 无损检测技术 中的应用; 定位抓取技术的应用以及分拣技术中的 应用。

\section{1 机器视觉系统在工业机器人零部件装配中 的应用}

在正常的工业生产和工业装备过程中, 一些零 部件的精准化和高效率都需要一定的操作基础, 而 这些操作基础几乎是人工难以完成的, 并且一些体 型巨大或者重量巨重的零部件, 往往都不是人力能 够进行移动和操作的, 都必须通过工业机器人进行 操作。因此在零部件总装备的行业应用范围内, 机 器人的视觉系统往往能够有效的帮助机器人在工作 中进行工作进度和效率的提升。目前国内相关学者 在研究工业机器人零件装配方面的操作, 通过利用 机械视觉系统, 对于零件的图像获取精准能力, 有 效地将工业机器人对你周围红资金范围极小的精确 性操作进行实现, 从而很大程度上完成了人工无法 完成的底座和减速器的智能装备, 这进一步的提高 了未来工业生产运行过程中零件装配中的智能化和 自动化, 有效的提升了整个工业生产的效率和精密 度。

\section{2 机器视觉系统在工业机器人无损检测技术 中的应用}

目前现有的无损检测技术一般情况下都具有处 理效率低, 精确度不高, 以及检查出库概率较低的 问题, 出现这些问题的原因, 从根本上来说主要来 源于人工检测。人工检测相比于工业机器人来说具 有不稳定性在检测过程中可能存在精确度不高, 实 有准确, 时而不准确的存在, 但是在工业机器人应 用上无损检测系统之后, 就完全能够满足现代工业 生产的基本检测条件。在通过机器视觉系统的情况 下, 将工业机器人与无损检测系统结合, 能够在生 产的过程中把检出工作有效的结合起来, 往往能够 有效的提高处理效率。而且机器人的无损检测系统
往往能够在不损坏零事件的前提下，通过一定的科 学技术和设备, 将检测物理内部结构进行分析和检 查, 相比于人工检测, 显得更加高效准确。工业机 器人存在的自动化能够大大提高无损检测的准确性 的同时, 也能够将整个车间的自动化程度提升, 将 智能技术全部运用于整个生产过程当中，把成品检 测和质量抽查等领域都涵盖进来。因此在机器视觉 系统下, 工业机器人把无损检测技术运用到生产车 间来, 能够有效地提升处理效率, 并且能够加大自 动化生产程度。

\subsection{1 包装检测}

在包装监测方面, 很大概率上会有大批量的检 查和测量, 例如检测印刷机是否能政策运行、辨别 产品包装上的字符和条码等, 机器视觉技术的应用 提升了产品的品质和可靠程度。金锋提出借助机器 视觉技术来辨别卷筒纸喷码, 来进一步确认喷码位 置所需要的图像检测算法和使用 SVM (Support Vector Machine, 支持向量机, 是使用频率较高的 一种判别方法）的点阵字符的分割、练习学习、辨 别算法。

\subsection{2 机械产品质量检测}

在机械产品的长度和品质检测方面, 机器视觉 监测技术被大范围使用。比如, 零件产品的几何参 数监测、在生产线的地点检测产品、检测产品表面 品质等。借助机器视觉技术检测, 和传统人工或传 统测量机械相比, 既提高了检测的精准度, 又提升 了工业自动化度。

王晓东等人根据已经掌握的机器视觉软件技术 加入了自己想法。根据机器精密度结合不同光源的 影响, 制造了精准型的计算测量与控制系统, 应用 能力非常强。

针对焊件外观图像的采集、处理以及识别等技 术的使用情况, 郭俊磊对此情况进行了深度的分析, 更深度的了解相关缺陷识别标准, 并且重新分析和 总结了焊接构件表面所具有的缺陷, 在知识库的基 础上研发专家系统, 以此来自动识别焊接构件表面 上所具有的问题。

\section{3 基于机器视觉的工业机器人定位抓取技术 的应用}

基于机器视觉的工业机器人的定位抓取技术主 要体现在产品的组装、零件的分拣以及汽车的生产 线上。

\section{3.1 产品组装}

基于机器视觉的工业机器人的定位和工业机器 人的技术可以能够帮助产品组装效率提升, 利用定 
位技术帮助产品迅速组装。在机器视觉的工业机器 人的参与之中可以帮助宫女做人员减轻工作压力, 利用机器视觉技术让电子机器人的系统可以不断的 在摄像头的基础上完成高难度的组装工作。产品组 装工人在组装产品之前可以先利用产品组装系统电 子技术先将产品零件的大小和尺寸输入到系统当中, 利用系统的记忆和系统的处理让系统可以在组装系 统中完成组装工作。

利用产品组装系统可以让工人在组装的过程之 中不断的进行机械操作, 可以节约工人在实际操作 之中的时间和精力。利用组装系统可以帮助组装系 统在动态和静态之中不断的进行转换, 提升机器组 装的速度。

\section{3.2 零件分拣}

基于机器视觉的工业机器人的定位系统可以帮 助零件的分拣工作。在定位系统的发展技术之下, 帮助工人完成相应的分工以及分拣的工作。零件的 分拣在实践之中是一项工作繁琐的事项, 利用定位 技术可以帮助提升工业产品的质量。在机器人的定 位技术的发展过程之中, 基于机器视觉的工业机器 人定位抓取系统可以通过一系列步骤来完成零件分 拣工作。与组装的系统一样, 分拣系统也是一项三 步骤发展的工作。首先也是信息录入系统, 在摄像 头的信息采集过程之中不断的利用摄像机拍摄零件 然后将图像传输至系统中。之后就是进行图像的风 不息处理, 让图像的技术可以在分拣技术之中得到 运用。最后也是最关键的效率环节, 利用分拣技术 的操作不断的将不同的零件进行分拣, 在实践之中 提升机器操作的效率。不断完善实践之中的零件识 别, 找出零件软件的缺陷。工人只需将零件图片传 输至系统中就可以让系统自行完成任务, 不仅提高了 工作效率还保证了零件分类的准确性。

\section{3.3 汽车生产线}

基于机器视觉的工业机器人可以让汽车生产从 手动变成自动化生产。在定位化技术的发展过程之 中, 利用信息化技术完成生产线的组装和汽车的焊 接、冲压涂装等工序工作。这样可以最大程度的提 高工业生产的效率, 完善汽车的生产线。以焊接工 序为例子, 焊接程序之中寻找焊接点是焊接程序之 中难度最大的工序。利用电子信息化技术可以帮助 在焊接工序之中完成最难的部分, 帮助焊接的更为 牢固。工人可以利用机器视觉的工业机器人定位抓 取系统来定位焊点然后在定位系统之中不断的进行 焊接点准确定位, 从而提升焊接的准确性和精确度。 除了焊接技术之外, 汽车的涂装工序最难的部分也 是均匀的喷漆, 利用电子信息化技术可以实现均匀 喷涂漆料。工人通过定位抓取系统定位可以实现均 匀喷涂以及避免漆料的浪费。

\section{4 基于机器视觉的工业机器人分拣技术分析}

工业机器人分拣系统主要是为了实现机器人的 自动化分拣工作。工业机器人分拣系统中的分拣技 术主要包括以下几点。分别是相机标定, 工件的识 别和定位, 工件识别, 工件定位以及工业机器人的 运用控制。

\section{4.1 相机标定}

在工业机器人分拣系统中, 利用机器的摄像头 技术可以进行物品的相机锁定。在相机锁定的基础 上进行工作带的相机有限锁定。在机器人的视觉基 础的技术发展之下, 利用机器人视觉技术进行相机 标定工作, 建立机器人的视觉发展空间位置坐标系 和图像坐标系。在空间位置体系的基础上进行视觉 定位, 通过视觉技术的分析来准确的判断工业机器 人以及目标物在图像之中的位置, 从而可以提升工 作效率。

\section{4.2 工件的识别和定位}

在整个电子信息化的系统运行过程中, 电子信 息化的技术在直接定位和发展的过程之中可以提升 机器人的操作质量, 配合图像匹配技术, 可以实现 多元化的匹配。例如工件的识别的匹配、特定区域 的匹配。其中, 特征匹配和灰度之间的依赖程度较 弱, 因而被广泛应用。

\subsection{3工件识别}

工件识别就是信息电子化技术之中的图像识别, 利用电子的图片匹配进行相应的数据比对和目标图 像的检索。利用电子信息化技术的已知模式, 在图 像数据库之中中找出相似的目标图像, 这样才可以 精确的进行位置的确定和方向的掌握。图像识别是 在模型数据库中获取相关的图像信息, 通过计算机 的图像特征比对进行相应的检测和匹配帮助图像识 别的目标准确定位。利用数据处理过的信息可以实 现相应的图像的识别工作，提升工作效率。

\section{4.4 工件定位}

工件识别可以帮助解决了在工业机器人分拣之 中的工作定位问题，利用分拣对象的信息化技术特 征, 可以准确的进行分拣对象信息的定位。工件定 位精确度的提高就需要对工件进行准确定位, 根据 数据库的图片比对可以进行分拣对象的位置的准确 确定。将数据库之中的图像坐标转化为数字坐标, 从而提高工件的分拣效率。

\section{4.5 工业机器人的运动控制}

在完成工件的检测、识别以及定位出相关工件 的坐标后, 要将机器人坐标系和图像坐标系进行转 
换，然后控制工业机器人的操作轨迹，使其能够完 成分拣任务, 更加准确地将目标工件放置在相应位 置。在工业机器人抓取工件或者物料的时候, 首先 必须要确定物料放置槽的坐标, 然后记录每个工件 的空间坐标所对应的全局变量。采用 $\mathrm{VC}++6$. 0 作为 软件编程语言, 编写机器人控制程序软件, 进而控 制工业机器人进行分拣操作国。

首先打开工业相机和启动拍照功能对工件或者 物料、场景等进行拍照, 然后借助图像处理模块和 坐标转化函数获得工件坐标, 由计算机向机器人发 布运行指示, 在工件由传送带传输到机器人下方时, 采用机械臂抓取或者吸取工件, 然后调转到物料放 置槽上方, 将工件放置其中, 进而实现物料或者工 件的分拣工作。

\section{3 结论:}

综合以上条件来看, 在机器视角下对工业机器 人技术进行提升, 能够有效的加强目前企业生产车 间的智能化和自动化程度。不仅如此, 很多人工不 能接触的领域, 往往工业机器人能够进行相应的检 测, 并且在具体数据进行分析的同时能够进行详细 的操作, 把真正的检测工作与生产工作做好。智能 技术能够普及于生产车间, 完全归功于工业机器人 技术的发展与提升, 所以在机器搜索下将工业机器 人本身的技术应用开发到生产这个建议及检测技术 当中, 能够有效的提升整体的企业经济能力, 从而 在一定程度上加强了国家的整体经济实力。

\section{REFERENCES}

[1] Chen Zhiguo, Xu Wenbo. Machine vision intelligent robot nondestructive testing system [J]. Computer engineering and application, 2009, 45 (14): 12-14

[2] Nie Bo. Research on industrial robot sorting technology based on machine vision [J]. Information and computer (theoretical Edition), 2018 (14): 104-106

[3] Wang Shuai, Xu Fang, Chen Liang, et al. Research on the application of vision technology in robot parts assembly [J]. Modular machine tool and automatic machining technology, 2015 (8): 107-110

[4] Xiao Zhuang, Wang Qiaohua, Wang Bin, et al. Grain size detection and grading method of red string fruit based on digital image and random least squares [J]. Food science, 2018, (15). 60-66

[5] Cao Gang. Concurrent engineering in metal shell modeling design. Selected ten-year excellent papers of China Industrial Design Association [M]. Beijing: China Light Industry Press, 1998.158-163

[6] Pan Yunhe, sun Shouqian, Bao Enwei. Development status and trend of computer aided industrial design [J]. Journal of computer aided design and graphics, 1999,11 (3): 248-252
[7] Wang Weiwei, Yu suihuai, Yang Gangjun, Yang Yanpu. Research on intelligent optimization model of constraint driven product form conceptual design [J]. China Mechanical Engineering, 2011,22 (15): 1770-1775

[8] Cheng Zhenbo, Ke Shanjun, Qin Yan. Learning behavior analysis of "computer aided industrial design" course under SPOC mode [J]. Journal of Southwest Normal University: Natural Science Edition, 2019,44 (9): 149-155

[9] Cai Bo, Lu Changde, Yu suihuai, Yang Haicheng. Top down geometric modeling of computer aided industrial design $[\mathrm{J}]$. Journal of computer aided design and graphics, 2001,13 (12): 1129-1132

[10] LV tie, Han Na. Intelligent Manufacturing: China 2025 -- Intelligent Manufacturing: global trend and China's strategy $[\mathrm{J}]$. People's forum · academic frontier, 2015,0 (11): 4-17

[11] Li zhekun, $\mathrm{Hu}$ Zhiyong, Li Yongping, Luo Minghong. Development of CIMS \&amp; CAID and information composition of CAID [J]. Journal of computer aided design and graphics, 2000,12 (11): $835-838$

[12] Zhu Shangshang, Luo Shijian, Zhao Jianghong. Research on modeling image scale of NC machine tools based on ergonomics [J]. Journal of computer aided design and graphics, 2000,12 (11): 873-875

[13] song Bo. Research on the training strategy of innovative high skilled talents under the background of "intelligent manufacturing" -- Investigation and analysis of talent demand in intelligent industrial robot related industries in Qingdao [J]. China training, 2018000 (005): p.11-12

[14] Tian Maosheng, Tang Xiaoqi, Meng Guojun, et al. Implementation of interactive control of industrial robot open control system based on Embedded PC [J]. Computer applications, 2010, (11). 3087-3090

[15] Wu Lihui, Zhao tie. In the application field of pathfinding robots for Buddha enterprises, the output value shows a blowout growth, and the robot high-end equipment industry accelerates the agglomeration [n]. Pearl River times, October 17, 2014 (4)

[16] Liu Hong, Liu Xiyu, Tang Qingming, John Hamilton Frazer. Computer aided design environment supporting innovative design of appearance modeling $[\mathrm{J}]$. Journal of computer aided design and graphics, 2003,15 (10): 1258-1262

[17] Li Bo, Yu suihuai, Chu Jianjie, Yang Yanpu, Du Wei, Du hemin. Research on computer aided system for product form design under multiple constraints [J]. China Mechanical Engineering, 2013,24 (3): 340-345 